\title{
CR Research Square \\ Spontaneous Space Closure After the Extraction of the Permanent First Molar on Mandibular Arch: A Retrospective Study
}

volkan Ciftci ( $\square$ volkangazi@yahoo.com )

Research article

Keywords: Extraction of Permanent Molar, Measurement Scale, Spontaneous space closure

Posted Date: January 16th, 2020

DOI: https://doi.org/10.21203/rs.2.21030/v1

License: (c) (i) This work is licensed under a Creative Commons Attribution 4.0 International License.

Read Full License 


\section{Abstract}

Background: First permanent molars (FPMs) with poor prognosis are generally extracted during the early formation of bifurcation of second permanent molars (SPMs) in order to achieve spontaneous space closure. The literature is limited about the factors associated with spontaneous space closure for mandibular arch. The aim of this study was to determine the prognostic radiographic factors, such as developmental stage of SPM, angulation of SPM and presence/absence of the third molar associated with the spontaneous space closure after the FPM extraction.

Methods: A total of 177 mandibular SPMs were included in this study. The prognostic factors determining successful space closure such as SPM developmental stage, SPM angulation using a measurement scale, and presence /absence of the third molar on the panoramic radiograph were evaluated.

Results: The mean age of the patients at the time of extraction of SPM was 9.4 years and post-extraction radiographic assessment at the time of the study was 12.7 years. Out of the total 177 mandibular SPMs, 36 SPMs (20.3\%) were at Demirjian stage D, $63(35.6 \%)$ at stage E, $60(34 \%)$ at stage F, $18(10.1 \%)$ at stage G. 16 SPMs (9\%) have distal angulation, 21 SPMs (11.8\%) have mesial angulation and 140 SPMs (79.1\%) have upright angulation. At the time of radiographic assessment, $80.2 \%$ of the mandibular quadrants showed evidence of third molar formation. Out of 177 mandibular SPMs, only 105 (59.3\%) exhibited successful space closure in the mandibular arch.

Conclusions: There is no statistically significance between the chronological age and the developmental stage of the mandibular SPM with the successful spontaneous space closure at the time of the dental extraction of FPMs. The presence of the mandibular third molar may be considered for the spontaneous space closure in mandibular arch.

Keywords : Extraction of Permanent Molar, Measurement Scale, Spontaneous space closure

\section{Introduction}

The first permanent molars (FPMs) erupt between 6 and 7 years of age in childhood and have a critical role on development of the dental occlusion [1,2]. Considering one of the first permanent teeth erupting as part of the growth and development, FPMs are more prone to the dental caries compare to the other permanent teeth [3-5]. A survey reported that FPM has been reported to be having the highest risk for dental caries in the permanent dentition [6]. Poor prognosis of FPM leading to dental extractions is affected by high prevalence of dental caries, large multiple surface restorations, pulpal symptoms, developmental defects such as molar-incisor hypomineralization $(\mathrm{MIH})$ [7-11].

Even early loss of FPMs have undesirable consequences, some studies report early extraction of FPMs may have favorable results from orthodontics perspective considering spontaneous space closure of FPMs [12-14]. Eruption of developing second permanent molars (SPM) may achieve to close mandibular 
FPM extraction space, before the chronological age of 8-9 years of the patients $[15,16]$. The United Kingdom national guidelines, which were updated within the last decade, have been advocated that early extraction of FPMs should be performed between the age of 8 and 10 years for the spontaneous space closure [17]. A recent review claimed that the time to extraction of FPM should be taken into consideration using the Demirjian classification for development of the SPM rather than by chronological age [12]. Similarly, Teo et al. highlighted the importance of the Demirjian classification for development of SPM rather than by chronological age [18].

The studies reported that spontaneous space closure would occur after the extraction of FPMs, yet with the differences about the favorable outcome of spontaneous closure for the FPM extraction space [1820]. Rahhal found that $84.6 \%$ of upper SPMs had spontaneous complete space closure after the extraction of FPMs [21]. The high rate of spontaneous space closure (94\%) for the maxillary arch was reported by Teo et al. [20]. Similarly, Patel et al. reported that $89.9 \%$ of maxillary quadrants exhibited successful space closure in their study population [19]. On the other hand, there are studies presenting conflicting results with less than $50 \%$ of successful space closure on the mandibular arch $[22,23]$. Studies reveal that spontaneous space closure occurs better in the maxilla compared to the mandibular arch. Spontaneous space closure was more difficult to achieve in the mandibular arch than in the maxillary arch $[9,20,24]$.

Factors affecting spontaneous space closure have been assessed in several studies $[12,18,19,22]$. The chronological age, developmental stage of SPMs, presence of third permanent molars, the direction of angulation of SPMs and premolars have been evaluated. Positive correlation was found between the spontaneous space closure and the chronologic age of the subjects [22, 25]. Teo et al. showed that mesial angulation of SPMs resulted in the most favorable spontaneous space closure after the FPM extraction [18]. Eichenberger et al. reported that third molar might have positive effect on spontaneous space closure in the mandibular arch [26].

Even if previous reports suggested that extraction of FPM when the bifurcation of the SPM is forming could be considered a predictor of successful space closure, there is still little evidence for the rationale of the timing of extraction of FPM. The data of literature about factors affecting spontaneous space closure is inadequate. The aim of this study is to investigate the prognostic radiographic factors such as (a) development stage of bifurcation of the SPM, (b) angulation of SPM and (c) presence of third permanent molar determining the spontaneous space closure after the early extraction of FPM.

\section{Materials And Methods}

The electronic dental records of 9580 pediatric dental patients who had a history of extraction of FPM between 2012-2018 at Cukurova University, Faculty of Dentistry were assessed by calibrated dental specialists (VC, AUG). Ethical approval for the study was obtained from Cukurova University, Faculty of Medicine Ethics Committee (Grant no: 50243401/2018-08). 
The inclusion criteria for the pediatric patients were as follows: patients age between 6 and 13 years old, pediatric patients whom having only unilateral or bilateral mandibular FPM extracted, patients having pre and post-extraction panoramic radiographs, complete eruption of SPMs and premolars in the postextraction panoramic radiographs. Pediatric patients having congenitally missing teeth, with special health care needs, history of unilateral or bilateral dental extraction of other permanent teeth, history of oral pathology such as odontoma, cyst, or surgical procedures, as well as pediatric patients who received orthodontic treatment during the follow-up were excluded from the study.

Out of 9580 pediatric patients' records, 133 pediatric patients with 177 extracted mandibular FPMs fulfilling the inclusion criteria were included to the final assessment of the study. The evaluation of the study was performed with the pre-extraction and post-extraction orthopantomographs of 133 pediatric patients. The following details, gender of the patients, age of the patients at the time of the extraction was recorded. Each patient's panoramic x-ray obtained at the time of FPM extraction was assessed for the stage of SPM development by using Demirjian Development Stage [27] where stage D defines the calcification of SPM crown, stage E defines the calcification of the bifurcation, stage $F$ defines the early root calcification, and stage $\mathrm{G}$ defines the late root calcification (Fig.1).

The angulation degree of the SPMs was measured by using the modified version of the Shiller method [28]. According to this method, an occlusal plane line (white line) passing through the occlusal plane of the FPM was determined. Another line passing through the midline of SPM was determined, and the distal angle was formed by the intersection of these two lines as the angulation of the SPM (Fig 2). A scale drawn on a transparent acetate paper of $5 \times 21 \mathrm{~cm}$ was used, as described by Patel et al. [19] to measure the degree of the angle. According to these angle measurements, the data were separated into three groups; Mesial, Upright, and Distal angled (M,U,D angulation) (Fig.3).The presence of developing third molar was considered with the assessment of presence of initial calcification or dental crypt formation of the third molars.

\section{Assessment of spontaneous space closure}

The assessment of space closure was described by evaluation of the space between the mesial aspect of the SPM and the distal aspect of the second premolar. Outcome of space closure was grouped into two groups; (a) successful space closure and (b) unsuccessful space closure (Fig.4). The presence of a visible contact between the SPM and the second premolar with no vertical or transverse discrepancy at the contact point was considered successful space closure (Fig.4.A). The presence of a visible space between the SPM and the second premolar was considered unsuccessful space closure (Fig.4.B). This space closure was determined whether SPM spontaneously closed the space after extraction of FPM via post-extraction panoramic radiographs. 


\section{Statistical analyses}

The SPSS package program (Version 23.0, Chicago, Illinois) was used for statistical analysis of the present data. Categorical measurements were summarized in terms of number and percentage, mean and standard deviation (continuous median and minimum-maximum where necessary). The Chi-square test or the Fisher test statistic was used to compare categorical variables. Distributions were checked when comparing continuous measurements between the groups, and the Student's t-test was used. Independent factors affecting the spontaneous space closure were determined by using stepwise regression analysis. The statistical significance level was 0.05 in all tests.

The study's retrospective power analysis yielded a power of 0.88 with a $95 \%$ confidence interval $(\mathrm{Cl})$. The pre-extraction panoramic radiographs of the pediatric patients were assessed by primary researchers (VC, AUG). The inter and intra-reliability of the examiners were assessed by Intraclass Correlation Coefficient (ICC). Initial panoramic radiographs were re-evaluated after two weeks and inter, and intra-reliability were estimated. Even the two examiners gave their independent assessments, in case of disagreement, the cases were re-evaluated, and a score was given after the consensus. ICC or the first rater and second rater showed good intra-agreement (Table 1).

\section{Results}

133 pediatric patients with 177 SPMs were evaluated for the prognostic radiographic factors of the spontaneous space closure on mandibular arch. Of those, 74 of them $(55.6 \%)$ were male and 59 of them $(44.4 \%)$ were female. The mean age of the patients at the time of the extraction of FPMs was 9.4 years and the mean time of assessment of post-extraction radiographs of the study was 12.7 years. Average follow-up duration between the pre-extraction and post-extraction radiographs was 3.5 \pm 1.1 years (2.7-6.0 years). Out of 177 SPMs, 105 SPMs (59.3\%) exhibited successful space closure. Table 2 shows the mean age of the pediatric patients at the time of the extraction according to the space closure.

The relation between spontaneous space closure and prognostic radiographic factors including SPM developmental stage, SPM angulation, and the presence or absence of third molars was evaluated. Out of 177 mandibular SPMs, 36 SPMs (20.3\%) were at Demirjian stage D, $63(35.6 \%)$ at stage E, $60(34 \%)$ at stage $F, 18(10.1 \%)$ at stage $G$ on assessment of pre-extraction panoramic radiographs. When the interaction between SPM development stage and space closure was analyzed, there was no statistically significance between SPM developmental stages and successful space closure $(p=0.749)$.

Out of 177 mandibular SPMs, 16 (9\%) SPMs have distal angulation, 21 (11.8\%) SPMs have mesial angulation and 140 (79.1\%) SPMs have upright angulation. The data were analyzed and there was no statistical significance between successful spontaneous space closure and SPM angulation $(p=0.220)$.At the time of pre-extraction orthopantomograph assessment, $80.2 \%$ of the quadrants showed evidence of third molar formation. The interaction between successful spontaneous space closure and presence or 
absence of the third molar was analyzed. For the mandibular arch, presence of the third molar was found statistically significant factor in the prediction of successful space closure $(p=0.034)$ (Table 3$)$.

The interaction between successful space closure and radiographic diagnostic factors (extraction age, SPM development stage, SPM angulation, presence/absence of developing third molar was also analyzed using regression analysis. Among these factors, presence of the third molar was found statistically significant as a predictor factor for successful space closure. Logistic regression analysis of all prognostic factors was shown in Table 4.

\section{Discussion}

The FPMs are on high risk for having dental caries due since they are one of the first permanent teeth to erupt, and to have developmental defects, like hypomineralization including $\mathrm{MIH}$. These teeth are commonly associated with short and long-term clinical dilemmas. In the literature, the incidence of developmental defects (hypomineralization)varies between $10 \%$ and $30 \%$ [12]. An report showed that primary indication for FPM extraction was extensive dental caries (70\%) [29]. Jalevik and Möller have reported that extraction of FPMs severely affected by MIH can be a good treatment alternative [9].

The studies showed that spontaneous space closure was in the range of $33.3 \%$ to $94 \%[18,19,24]$. The similar studies also reported the differences in the amount of space closure between the maxilla and mandible and it was found that spontaneous space closure was more likely to be achieved in the maxilla than the mandible after dental extraction of FPMs $[9,20,24]$. Teo et al. evaluated spontaneous space closure after dental extraction of FPMs among patients aged 7-13 years. They found that $66 \%$ of the extraction space of FPMs on mandible was closed completely after five years of post-extraction [20]. Patel et al 19. reported that $49 \%$ of 153 mandibular SPMs had a successful space closure compatible with previous finding of rate of $38.5 \%$ and $50 \%$ for the mandibular arch $[22,23]$. In the present study, the FPM was extracted at the mean age of 9.4 years and $59.3 \%$ of the 177 quadrants had a successful spontaneous space closure.

Teo et al. [20] have highlighted the importance of the developmental stage of the SPMs and reported that stage $\mathrm{E}$ of the Demirjian classification (early bifurcation stage) might be the ideal time for the extraction of the FPMs. Gill et al. explained that extraction of the mandibular FPM before or after early bifurcation stage will not result in complete space closure, resulting in distal drifting, tilting and rotation of unerupted premolar [30]. In 2016, Patel et al. found that there was no relationship between the developmental stage of SPM and dental age considering the spontaneous space closure in the mandibular arch [19]. In this study, no significant relationship was observed between the developmental stage of SPM and spontaneous space closure $(p=0,749)$. These results are compatible with findings of study by Patel et al. Furthermore, Patel et al. shown that the angulation of SPMs was a predictor factor in the spontaneous space closure [19]. According to Patel et al., the angulation of the developing SPM has a statistically significant relationship with space closure in the mandibular arch. In a recent study of Teo et al. [18], it was evaluated that the spontaneous space closure from panoramic radiographs in a group of children 
with the mean age of 9.2 years at the time of FPM dental extraction. They showed that the mesially angulated SPM in relation to the FPM could result in most favorable outcomes. Yet, in this study, it was not found a significant relationship between SPM angulation and spontaneous space closure for the mandibular arch. In this study, majority of the SPM angulation was found upright (84.7\%).

Initial crypt formation of the mandibular third molar has been begun to develop at $9 \pm 2.35$ years of age in girls and $9.79 \pm 1.63$ in boys [31]. In the literature, the prevalence of impacted third molar has been reported to be $24.4 \%$ [32]. Elsey and Rock reported that up to $73 \%$ of third molars were graded as impacted in European young adults [33]. A few studies have shown that mandibular FPM extraction increases the available space for mandibular third molar eruption and helps third molars move into a better position [30, 34, 35]. Yavuz et al. evaluated the effects of FPM extraction on development and eruption of the third molar in adolescents. They found that of $17 \%$ third molars had erupted on the FPM extraction quadrants comparing to only $6.6 \%$ of third molars on the non-extraction quadrants [36]. Ay et al. observed that extracting FPM teeth can provide the eruption for third molars by mesial movement of the molars [35]. This is due to fact that developing and eruption force of the third molars on the FPM extraction side may accelerate to drift of SPM into the extraction space of FPM in our study population. In this study, when a statistical model using regression analysis was undertaken to determine the factors affecting the success of spontaneous space closure, the presence of third molar was found to be statistically significant among all the variables. A recent systematic review included observational and retrospective studies mentioned that the consequences of FPM extraction include a decrease in post extraction space, via development and eruption of SPM and presence of third molar [12]. Eichenberger et al. [26] also agreed that the presence of developing third molar could have a positive effect on spontaneous space closure compatible with our data in the mandibular arch.

\section{Limitations}

This retrospective study has some limitations, such as spontaneous space closure was evaluated radiographically after the FPM extraction. Also, our data did not provide any information about how the space was closed in terms of bodily or through tipping of SPM or premolar. Further researches are needed to assess the factors such as skeletal and dentoalveolar relationships including cephalometric radiographs or dental models to comparing with pre-extraction and post extraction.

\section{Conclusions}

1. The consequences of early extraction of FPM include a decrease in post extraction space.

2. The developmental stage of SPM was not found to be statistically significant for the spontaneous space closure in the mandibular arch.

3. The angulation of the SPM has no relationship to the spontaneous space closure in the mandibular arch. 
4. Developing of third molar should be considered for spontaneous space closure following FPM extraction.

\section{Declarations}

\section{Abbreviations}

FPM: First permanent molar; SPM: Second permanent molar; MIH: Molar-incisor hypomineralization; Cl: Confidence interval; ICC: Intraclass correlation coefficient; OR: Odds ratio

\section{Acknowledgements}

We would like to acknowledge the help of Cagla Sariturk for statistical analysis of the study. We would also like to thank Dr. Roland Blankenstein for kindest support.

\section{Availabitiy of data and materials}

Raw data of the study have been obtained from Cukurova University, Faculty of Dentistry. Statistical data are available upon request to the corresponding author.

\section{Funding}

This research did not receive any specific grant from funding agencies in the public, commercial, or notfor-profit sectors.

\section{Authors' contributions}

VC investigated the idea of the study, developed the protocol for the study. VC, AUG and CD carried out the literature search and data extraction. VC, AUG were involved in data analysis and interpretation of the data. IY, FS and were involved in the study design and drafting of the manuscript. AHT critically reviewed the manuscript. All the authors revised and approved the final manuscript

\section{Ethics approval and consent to participate}

Ethical approval for this study was obtained from Cukurova University, Faculty of Medicine Ethics Committee (Grant no: 50243401/2018-08).

\section{Competing interests}

The authors declare that they have no competing interests.

\section{References}


1.Angle EH. Treatment of Malocculsion of the Teeth: SS White dental manufacturing Company; 1907.

2.Anatomy O. Histology and Embryology: BKB Berkowitz, GR Holland, BJ Moxham. Mosby; 2002.

3.Skeie M, Raadal M, Strand G, Espelid I. The relationship between caries in the primary dentition at 5 years of age and permanent dentition at 10 years of age-a longitudinal study. Int J Paediatr Dent. 2006; 16(3):152-60.

4.Pitts N, Chestnutt I, Evans D, White D, Chadwick B, Steele J. 1 Verifiable CPD Paper: The dentinal caries experience of children in the United Kingdom, 2003. Br Dent J. 2006; 200:313-320.

5.Batchelor PA, Sheiham A. Grouping of tooth surfaces by susceptibility to caries: a study in 5-16 yearold children. BMC Oral Health. 2004; 4 (1):2.

6.Todd JE, Dodd T. Children's Dental Health in the United Kingdom, 1983: A Survey Carried Out by the Social Survey Division of OPCS, on Behalf of the United Kingdom Health Departments, in Collaboration with the Dental Schools of the Universities of Birmingham and Newcastl: Stationery Office; $1^{\text {st }}$ edition, 1985.

7.Vargas-Ferreira F, Salas M, Nascimento G, Tarquinio S, Faggion Jr C, Peres M, et al. Association between developmental defects of enamel and dental caries: A systematic review and meta-analysis. $J$ Dent. 2015; 43(6):619-28.

8.Balmer R, Toumba J, Godson J, Duggal M. The prevalence of molar incisor hypomineralisation in Northern England and its relationship to socioeconomic status and water fluoridation. Int J Paediatr Dent. 2012; 22(4):250-257.

9.JÄlevik B, MÖller M. Evaluation of spontaneous space closure and development of permanent dentition after extraction of hypomineralized permanent first molars. Int J Paediatr Dent. 2007; 17(5):328-335.

10.Mathu-Muju KR, Kennedy DB. Loss of Permanent First Molars in the Mixed Dentition: Circumstances Resulting in Extraction and Requiring Orthodontic Management. Pediatr Dent. 2016; 38(5):46-53.

11.Ong DV, Bleakley J. Compromised first permanent molars: an orthodontic perspective. Australian Dent J. 2010; 55(1):2-14.

12.Saber AM, Altoukhi DH, Horaib MF, El-Housseiny AA, Alamoudi NM, Sabbagh HJ. Consequences of early extraction of compromised first permanent molar: a systematic review. BMC Oral Health. 2018; 18(1):59.

13.Penchas J, Peretz B, Becker A. The dilemma of treating severely decayed first permanent molars in children: to restore or to extract. J Dent Child. 1994; 61(3):199-205. 
14.El Sheikh M, Ali A. Planned extraction of first permanent molars during late childhood: a clinical note and mini-review. Dent Oral Craniofac Res. 2015; 1(3):77-80.

15.Cobourne MT. Orthodontic Management of the Developing Dentition. Springer; 2017.

16.Thunold K, editor Early loss of the first molars 25 years after. Rep Congr Eur Orthod Soc. 1970.

17.Cobourne M, Williams A, McMullan R. A guideline for the extraction of first permanent molars in children. London: Royal College of Surgeons of England, 2009.

18.Teo TK-Y, Ashley PF, Derrick D. Lower first permanent molars: developing better predictors of spontaneous space closure. Eur J Orthod. 2015; 38(1):90-95.

19.Patel S, Ashley P, Noar J. Radiographic prognostic factors determining spontaneous space closure after loss of the permanent first molar. Am Journal Orthod Dentofacial Orthop. 2017; 151(4):718-726.

20.Teo T, Ashley P, Parekh S, Noar J. The evaluation of spontaneous space closure after the extraction of first permanent molars. Eur Arc Paediatr Dent. 2013; 14(4):207-212.

21.Rahhal AA. Extraction Timing of heavily destructed upper first permanent molars. Open J Stomatol. 2014;4(3):161.

22. Thilander B, Skagius S, editors. Orthodontic sequelae of extraction of permanent first molars. A longitudinal study. Rep Congr Eur Orthod Soc; 1970.

23.Plint $D$, editor The effect on the occlusion of the loss of one or more first permanent molars. Rep Congr Eur Orthod Soc; 1970.

24.Rãducanu AM, Feraru V, Herteliu C, Rãducanu MA. Prevalence of loss of permanent first molars in a group of Romanian children and adolescents. Oral Health Dent Manag. 2009; 8(3):3-10.

25.Cobourne M, Williams A, Harrison M. National clinical guidelines for the extraction of first permanent molars in children. Br Dent J. 2014; 217(11):8-643.

26.Eichenberger $M$, Erb J, Zwahlen $M$, Schätzle $M$. The timing of extraction of non-restorable first permanent molars: a systematic review Introduction. Eur J Paediatr Dent. 2015; 16(4):8-272.

27.Demirjian A, Goldstein H, Tanner J. A new system of dental age assessment. Human biology. 1973; 45(2):211-227.

28.Shiller WR. Positional changes in mesio-angular impacted mandibular third molars during a year. The J Am Dent Assoc. 1979; 99(3):460-464.

29.Albadri S, Zaitoun H, McDonnell S, Davidson L. Extraction of first permanent molar teeth: results from three dental hospitals. Br Dent J. 2007; 203(7):14. 
30.Gill D, Lee R, Tredwin C. Treatment planning for the loss of first permanent molars. Dental update. 2001; 28(6):304-308.

31.Roberts G, Parekh S, Petrie A, Lucas V. Dental age assessment (DAA): a simple method for children and emerging adults. Br Dent J. 2008; 204(4):7.

32.Carter K, Worthington S. Predictors of third molar impaction: a systematic review and meta-analysis. J Dent Res. 2016; 95(3):76-267.

33.Elsey M, Rock W. Influence of orthodontic treatment on development of third molars. Br J Oral Maxillofac Surg. 2000; 38(4):3-350.

34.Bayram M, Özer M, Arici S. Effects of first molar extraction on third molar angulation and eruption space. Oral Surg Oral Med Oral Pathol Oral Radiol Endod. 2009; 107(2):14-20.

35.Ay S, Ağar Uu, Bıçakçı AA, Köşger HH. Changes in mandibular third molar angle and position after unilateral mandibular first molar extraction. Am J Orthod Dentofacial Orthop. 2006; 129(1):36-41.

36.Yavuz Ib, Baydaş B, İkbal A, Dağsuyu iM, Ceylan Is. Effects of early loss of permanent first molars on the development of third molars. Am J Orthod Dentofacial Orthop. 2006; 130(5):634-8.

\section{Tables}

Table 1. Intraclass correlation co-efficient (ICC)

\begin{tabular}{|c|c|c|c|}
\hline$r$ & $\% 95$ G.A. & F & $p$ \\
\hline 0,811 & $0,79-0,83$ & 107,63 & 0,0001 \\
\hline
\end{tabular}

Table 2. Mean and standard deviations of extraction age and post-extraction according to the space closure

\begin{tabular}{|l|c|c|c|c|c|}
\hline & \multicolumn{4}{|c|}{ Spontaneous Space Closure } & \\
\hline & \multicolumn{2}{|c|}{ Successful(n=105) } & Unsuccessful(n=72) & \\
\hline & Mean \pm SD & Min-Max & Mean \pm SD & Min-Max & $\mathrm{p}$ \\
\hline Chronological Age (years) & $9,5 \pm 1$ & $6,1-10,6$ & $9,3 \pm 0,9$ & $6,9-10.8$ & 0,703 \\
\hline
\end{tabular}

Table 3.The correlation between spontaneous space closure and prognostic radiographic factors 


\begin{tabular}{|c|c|c|c|c|c|}
\hline & \multicolumn{4}{|c|}{ Spontaneous SpaceClosure } & \multirow[b]{3}{*}{$p$} \\
\hline & \multicolumn{2}{|c|}{ Successful } & \multicolumn{2}{|c|}{ Unsuccessful } & \\
\hline & $n$ & $\%$ & $n$ & $\%$ & \\
\hline \multicolumn{6}{|c|}{ Demirjian Development Stage } \\
\hline Stage D & 22 & 21.0 & 14 & 19.4 & 0.749 \\
\hline Stage E & 36 & 34.3 & 27 & 37.5 & \\
\hline Stage $\mathrm{F}$ & 34 & 32.4 & 26 & 36.1 & \\
\hline Stage $\mathrm{G}$ & 13 & 12.4 & 5 & 6.9 & \\
\hline \multicolumn{6}{|c|}{ SPM angulation } \\
\hline Distal & 10 & 9.5 & 6 & 8.3 & 0.220 \\
\hline Mesial & 16 & 15.2 & 5 & 6.9 & \\
\hline Upright & 79 & 75.2 & 61 & 84.7 & \\
\hline \multicolumn{6}{|c|}{ 3.molar absence/presence } \\
\hline Absence & 15 & 14.3 & 20 & 27.8 & $0.034^{*}$ \\
\hline Presence & 90 & 85.7 & 52 & 72.2 & \\
\hline
\end{tabular}

$* \mathrm{p}<0.05$

Table 4: Logistic regression analysis of prognostic radiographic factors

\begin{tabular}{|c|c|c|c|c|c|c|c|c|}
\hline & B & S.E. & Wald & df & $p$ & OR & \multicolumn{2}{|c|}{$95 \%$ CI for OR } \\
\hline & & & & & & & Lower & Upper \\
\hline Extraction age & .16 & .18 & .81 & 1 & .368 & 1.175 & .83 & 1.67 \\
\hline Demirjian & .34 & .39 & .74 & 1 & .389 & 1.403 & .65 & 3.03 \\
\hline$@ 2^{\text {nd }} \cdot \operatorname{molar}(\mathrm{U})$ & & & 2.45 & 2 & .294 & & & \\
\hline$@ 2^{\text {nd }} \cdot \operatorname{molar}(\mathrm{D})$ & .02 & .59 & .001 & 1 & .972 & .980 & .31 & 3.10 \\
\hline$@ 2^{\text {nd }} \cdot \operatorname{molar}(\mathrm{M})$ & .88 & $.564-$ & 2.44 & 1 & .118 & .414 & .14 & 1.25 \\
\hline$@ 3^{\text {rd }} \cdot \operatorname{molar}(\mathrm{P})$ & .89 & .41 & 4.79 & 1 & .029 & 2.435 & 1.10 & 5.40 \\
\hline Constant & .75 & 1.82 & .17 & 1 & .678 & .470 & & \\
\hline
\end{tabular}

a. Variable(s) entered on step 1: Extraction age, Demirjian Stage, @ Second molar angulation (M=Mesial,D=Distal, U=Upper), @ Presence of third molar ( $\mathrm{P}=$ Presence $)$. 
Figures

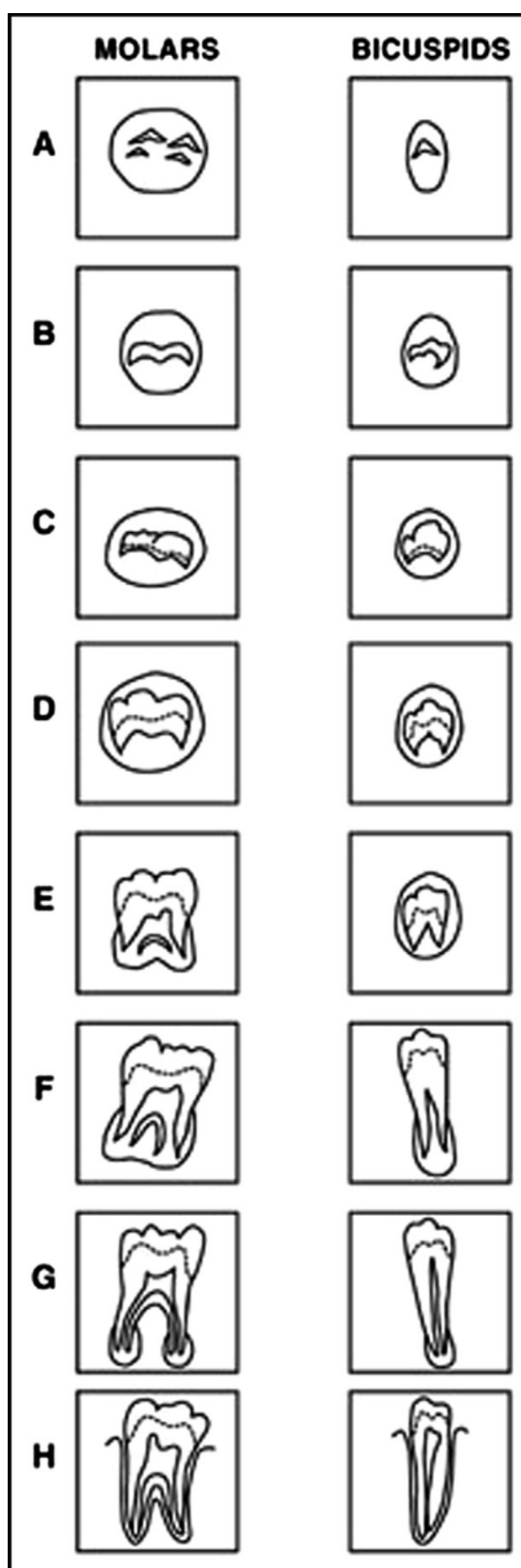

Figure 1 


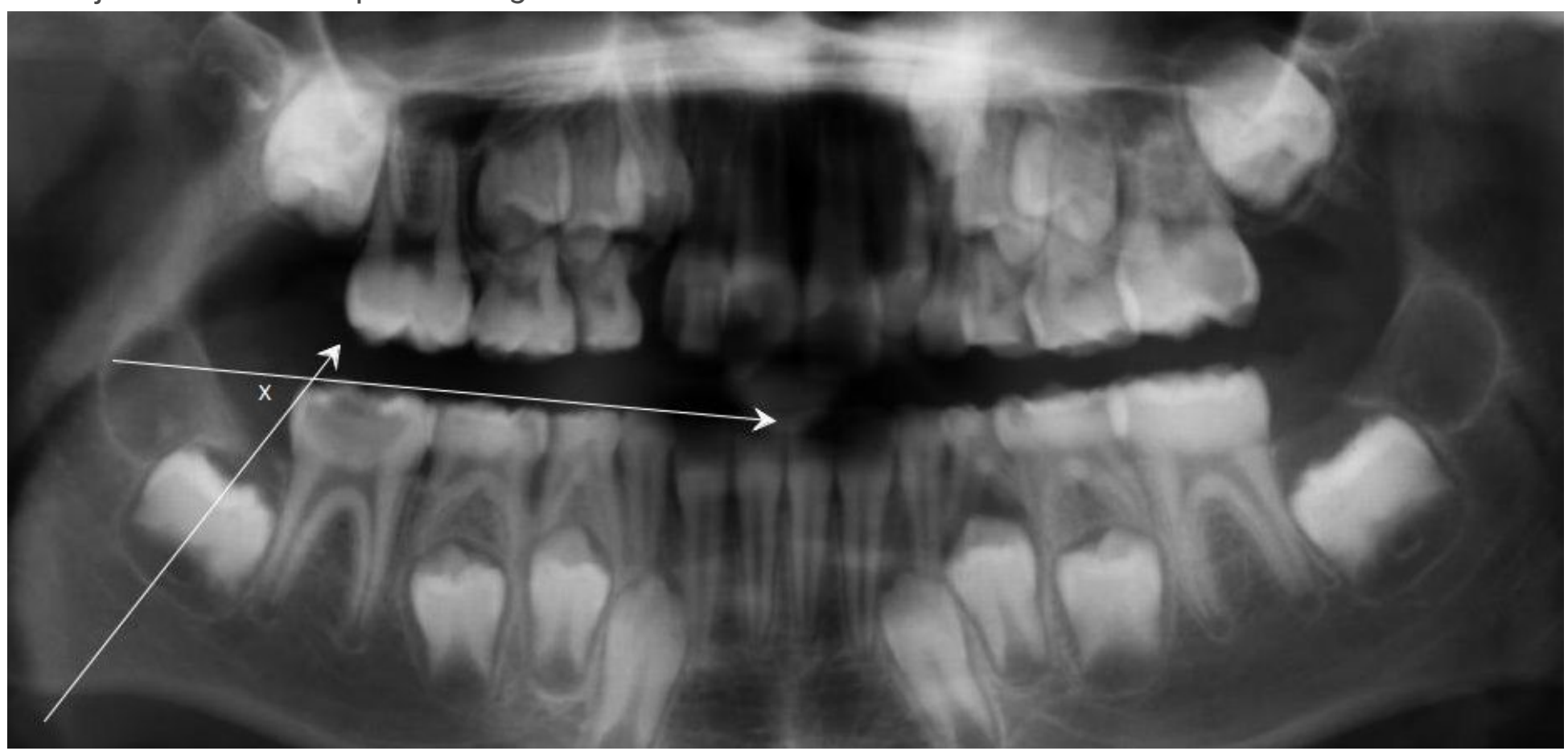

Figure 2

Drawing panoramic film showing the lines and angles for angular measurements.

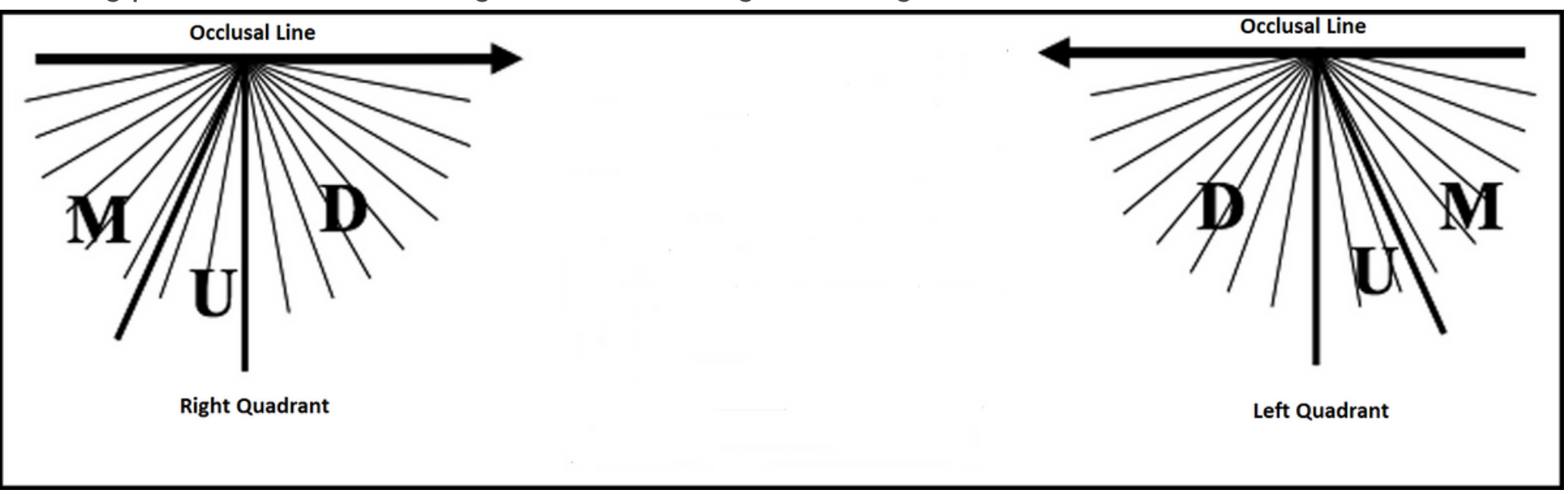

\section{Figure 3}

The measurement scale described by Patel et al.13 to determine the SPM angulation 


\section{Figure 4}

A- Visible radiologic contact-contact point between SPM and second premolar B- Visible radiologic space (non-contact) between SPM and second premolar

\section{Supplementary Files}

This is a list of supplementary files associated with this preprint. Click to download.

- Ethics.pdf 